Majority of dental

\section{professionals feel that}

\section{patients have been}

\section{understanding of changes during the pandemic}

The majority of dental professionals surveyed stated that patients have been understanding of the changes that have been made during the pandemic, according to new research published by the Dental Defence Union (DDU).

The DDU surveyed 538 dental members about their working circumstances during the pandemic and found that while $95 \%$ said patients were understanding, this has not alleviated concerns within dentistry. The DDU found that $60 \%$ of respondents were concerned about facing a complaint, claim or GDC investigation relating to the pandemic. Other key findings included $55 \%$ believing that remote consultations will remain in place following the end of the pandemic. Additionally, 59\% also found it easy to stay up-to-date with the latest guidance issued during the pandemic.

Leo Briggs, deputy head of the DDU commented: 'Dental professionals are used to dealing with stressful, high pressure situations. Nevertheless, the pandemic has heightened some existing challenges while presenting many new ones.

'Working practices have dramatically changed during the pandemic and it is good that the majority of patients have been understanding. However, it's clear that dental professionals are concerned about the risk of a future complaint or claim as a result of treatment undertaken or postponed during the pandemic.'

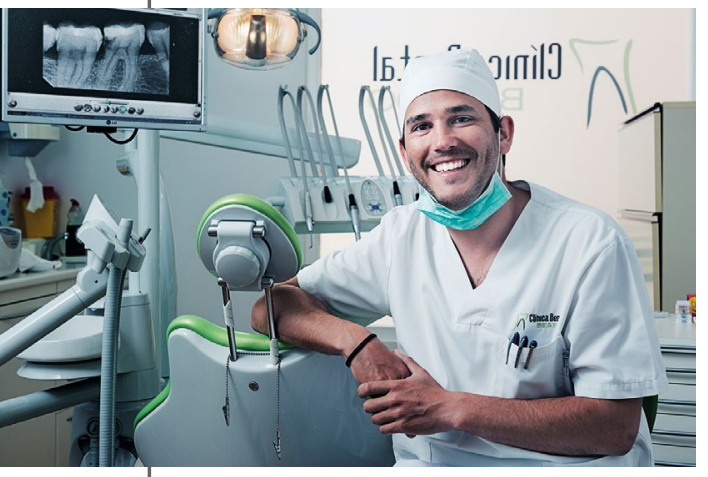

\title{
Lockdown forces Brits to become 'DIY dentists'
}

The COVID-19 pandemic has had a severe impact on the UK's oral health, according to the findings of new research. ${ }^{1}$

An investigation by the Oral Health Foundation and Colgate shows that almost one-in-five (18\%) have not seen a dentist for over two years, not just because of lockdown.

Meanwhile, more than one-in-three (36\%) have reported toothache during COVID-19, with nearly one-in-ten (9\%) experiencing the pain for longer than two weeks.

To remedy this, a concerning number of patients are going online to find solutions to fix their own dental problems. The findings show almost one-in-four (24\%) have been online to fix oral health problems at home.

Chief Executive of the Oral Health Foundation, Nigel Carter OBE, believes more must be done to educate and motivate patients to prioritise their oral health.

Dr Carter says: 'A worrying number of patients have neglected their oral health during the pandemic. This could have potentially lasting consequences, not only for the health of their mouth but for their general wellbeing also.

'The public's awareness of what causes dental caries and how to prevent it, remains poor. Yet, preventing oral diseases like caries is completely in their own hands. All it takes is the correct care.'

To help patients identify their risk of dental caries and help them prevent it, the Oral Health Foundation has launched a new online hub - The Truth About Tooth Decay with the support of Colgate.

The area includes guidance on how the dental team can help improve a patient's awareness of caries, along with strategies for preventing caries, especially in moderateto-high risk patients.

There is also advice about some of the treatments available, such as high fluoride toothpastes, and information about remote consultations and triage.

Scientific Affairs Project Manager at Colgate Emanuele Cotroneo, highlights the importance of the new online resource.

Dr Cotroneo says: 'As leader in oral care, Colgate has a fundamental role to play in improving people's oral health. Colgate exerts this role by means of technological innovations and by partnering with the

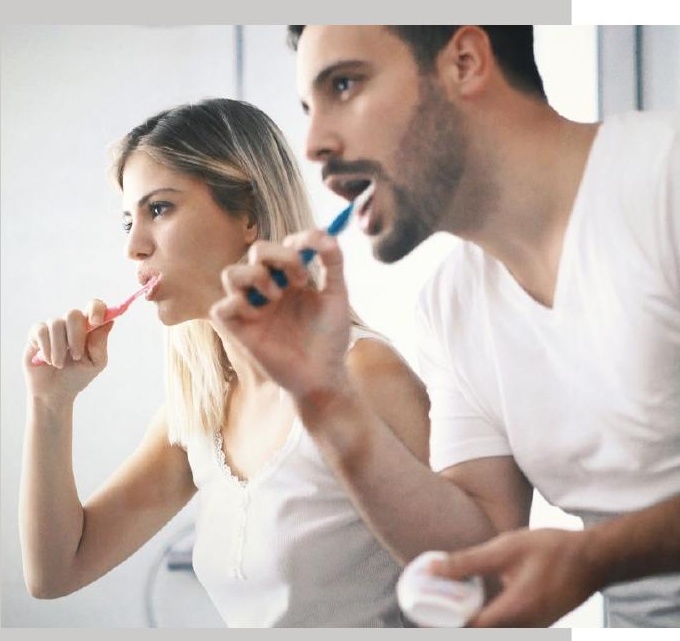

dental profession in ways that help them to meet the needs of their patients.

'The collaboration with the Oral Health Foundation is an example of Colgate's commitment to its role in improving oral health. It is clear from this survey that these challenging times have affected the oral health habits of the UK population.

'Colgate reinforces the message from the Oral Health Foundation that oral health should not be neglected as it is a crucial part of your wellbeing and tightly linked with overall health'

'Most of us in practice would have experience with a patient who has attempted DIY dentistry', adds Dr Carter. 'We are extremely worried by the number of people now attempting their own dental solutions at home. That's why our aim with this new campaign is not only to raise awareness of dental caries but to help drive more people back into the dental practice.

'Our findings tell us the people are still uncertain access to dentistry and about the safety of dental practices during COVID19. We are here to reassure patients, and the general public who might not attend checkups regularly, that dentistry is a safe place to go and that any problems should be dealt with by a trained and qualified professional.'

The Truth About Tooth Decay can be found at www.dentalhealth.org/ thetruthabouttoothdecay.

\section{Reference}

1. Oral Health Foundation and Colgate-Palmolive (2021) 'Dental Caries Awareness Survey', UK, Broadcast Revolution, December 2020, Sample 2,008 